\title{
DOES PITYRIASIS ROSEA KOEBNERISE?
}

\author{
by Nwabudike Lawrence Chukwudi
}

comment:

Ass. Prof. Antonio Chuh MD FRCP FRCPCH

School of Public Health, The Chinese University of Hong Kong, Prince of Wales Hospital, Shatin, Hong Kong

Prof. Vijay Zawar MD DNB DV\&D FAAD

Department of Dermatology, Godavari Foundation Medical College and Research Center, DUPMCJ, India

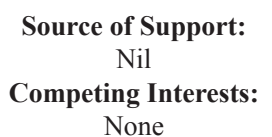

Our Dermatol Online. 2013; 4(2): 191

Date of submission: 21.03.2013 / acceptance: 28.03.2013
We read with admiration the article by Nwabudike on possible association of pityriasis rosea (PR) and Köebner/ isomorphic response (IR) [1]. A 35-year-old female exhibited PR lesions at bilateral cubital fossae, presumably related to venepuncture. The author believes that there exists no previous report on such association.

We note a typical PR lesion over a superficial vein at the left cubital fossae (Figure 1, [1]). However, the PR lesion at the right cubital fossa was not directly riding on or adjacent to the superficial veins (Figure 2, [1]). A venepuncturer viewing an obvious superficial vein over the radial aspect of the fossa and a Y-shaped thick superficial vein over the ulnar aspect would unlikely puncture at the PR lesion site shown which was near the centreline of the fossa. Should this be the case, IR can be established on one site only - the left arm, which might be coincidental. We therefore cast doubts on whether this patient is genuinely exhibiting IR at the backdrop of venepuncture. However, there exists a possibility that the venepuncturer might have selected a deep vein which was not captured in Figure 2 [1], and we therefore trust the judgements of the author.

We also wish to point out that IR was suspected to occur in a 41-year-old male patient with both PR and secondary syphilis [2]. (However, such might be termed isotopic response in the modern terminology $[3,4]$, as rightly pointed out by the author). IR has also been reported in a patient with inversePR [5]. The author may have just inadvertently omitted these reports in his literature search.

Four types of IR have been described - true IR (frequent development of lesions in traumatised and previously uninvolved skin, e.g. psoriasis), pseudo IR (auto-inoculation of microbes, e.g. viral warts and molluscum contagiosum), occasionally-occurring IR (infrequent development, e.g. Darier's disease and erythema multiforme), and questionable IR (limited case reports, e.g. morphoea) [6]. At the current state of knowledge, we believe that PR might be associated with questionable IR only. Should there be further reports, such association might be occasional IR if the pathogen is yet unidentified, or pseudo IR if we finally identify the true culprit(s) in PR, with the latter scenario being highly unlikely at this moment, considering uncertain microbiological aetiology of PR [7].

\section{REFERENCES}

1. Nwabudike LC: Does pityriasis rosea Köebnerise? Our Dermatol Online. 2013;4:189-190.

2. Secher L, Weismann K, Kobayasi T: Pityriasis rosea eruption in secondary syphilis: an isomorphic phenomenon? Cutis. 1985;35:403-4.

3. Wolf R, Wolf D, Ruocco E, Brunetti G, Ruocco V: Wolf's isotopic response. Clin Dermatol. 2011;29:237-40.

4. Wolf R, Lotti T, Ruocco V: Isomorphic versus isotopic response: data and hypotheses. J Eur Acad Dermatol Venereol. 2003;17:1235.3.

5. Gibney MD, Leonardi CL: Acute papulosquamous eruption of the extremities demonstrating an isomorphic response. Inverse pityriasis rosea (PR). Arch Dermatol. 1997;133:651,654.

6. Boyd AS, Neldner KH: The isomorphic response of Köebner. Int J Dermatol. 1990;29:401-10.

7. Chuh A, Zawar V, Sciallis G, Law M: Gianotti-Crosti syndrome, pityriasis rosea, asymmetrical periflexural exanthem, unilateral mediothoracic exanthem, eruptive pseudoangiomatosis, and papular-purpuric gloves and socks syndrome - succinct reviews and arguments for a diagnostic criteria. Infect Dis Rep. 2012;4:38-48. 